\title{
The Appraisal Procedure For The Importance Of Node in Corporate Marketing Network
}

\author{
Junquan Gong ${ }^{a}$ *, Xiaohong Qin ${ }^{b}$ \\ Tianshui Normal University School of Business Tianshui Gansu china 741001 \\ a15291857968@139.com, bqinxiaohong12@sohu.com
}

\begin{abstract}
Keywords: Corporate marketing network; robustness; uncertainty; node importance
Abstract: Marketing network is a complicated adaptive system, in which there are many uncertain factors. The work to strengthen the protection for important node in marketing network is a decisive factor for network marketing to function normally in product marketing. In this thesis, the appraisal procedure for the importance of network node in marketing is mooted, at the same time, its effectiveness is proved by examples. To identify the important node in marketing network and improve robustness of marketing network, this research conclusion has certain value in practice.
\end{abstract}

\section{Introduction}

Corporate marketing network is a series of interrelated product distribution process which involved the collection of interdependent organizations or institutions [1]. With the development of global economic integration and information technology, corporate marketing network coverage has become increasingly broad structure more complex, global marketing network of multinational organizations and institutions everywhere formation [2]. In the corporate marketing network, each network member (organization or agency) shall be deemed an act subject, these actors with independent judgment and behavior, at the same time, each of the network members will act in accordance with other network members as well as changes in the environment constantly modify their behavior to adapt to the environment and corporate marketing [3]. Thus, corporate marketing network is a complex adaptive system, it will emerge a number of individual organizations or agencies do not have the characteristics. For enterprise network marketing a complex system like this, there are many uncertainties, there is disturbance uncertainties from the network and the inner workings of the uncertainty of the external environment. This disturbance, from the dangers of perspective, the impact of the operation and efficiency of small business marketing networks, large enterprise marketing network is spread along the link (marketing channels), resulting in partial or total loss of function of the marketing network (network collapse). Based on the uncertainty analysis of enterprise marketing network, based on network topology based enterprise marketing given the importance of corporate marketing network node assessment methods, and through the calculations to verify the validity of the assessment methods.

\section{Uncertainty Corporate Marketing Network}

With economic globalization and market competition intensifies, the modern enterprise marketing system is increasingly complex. Manufacturers through a complex production process, to produce the final product, and then through wholesalers, retailers and other intermediaries to deliver products to the end user or consumer. In the corporate marketing network, there are various forms of uncertainty.

Sources of uncertainty enterprise marketing network can be divided into two categories: the uncertainty of the inner workings of uncertainty and external environment [4]. After the corporate marketing network by uncertainties interference, there will be two kinds of situation in general[5]. A case is the marketing network members can not adapt to business marketing system, its capacity can not match after interference environment, such as poor management, self-collapse, etc., so that you lose all the other nodes in the network convergence, this interference is actually only affects to other network members in touch with it, that the network members became isolated point, disconnect all 
connections to other network members, it reflected to the point in the network; another case is unable trading occurs between Wang Yuan such as transportation routes interrupted transaction can not be reached, etc., so that makes the existence of trading relations Wangyuan break contact, embodied in the network to go to the edge. Suppose, if for some reason a business marketing network marketing channel upstream node can not supply on time delivery to the end customer will be forced to cancel, these uncertainties will force manufacturers, wholesalers, retailers increase inventory, when the company marketing system presents a mesh structure, the problem is more complex, the uncertainty will rapidly spread like a plague across the marketing network. Therefore, the strengthening of corporate marketing network in an important node protection is an important means of corporate marketing network management.

\section{The Method of Importance Node Evaluation of Corporate Marketing Network}

Taking full account of corporate marketing network topology and the formation process based on the paper document [6] node shrink method mentioned in some degree of improvement, in order to assess the importance of corporate marketing network marketing network members, the most Wang Yuan is important after a complex network of corporate marketing shrinkage allows companies marketing complex network nodes represent the greatest degree of aggregation of network members [7]. According to this method, through a complex network of corporate marketing in each node shrinkage, and ultimately to determine the order of importance of corporate marketing network all network members to find business marketing network in key network members, by strengthening the protection of critical Wang Yuan and management to improve the robustness of corporate marketing network.

Refers to a contraction of the node $v_{i}$ and the node $k_{i}$ connected to the node $v_{i}$ associated with the integration, by a new node to replace the node $k_{i}+1$, they are attached edges of the original are now connected to the new node [3]. The right side is the right value for the contraction nodes and peripheral nodes and edges even if multiple paths to shrink form the shortest distance between nodes.

The definition of corporate marketing complex network aggregation degree:

$$
\partial(G)=\frac{1}{S \times L(G)}
$$

In the formula (1), $S$ represents a complex network of corporate marketing and the right of the average point, , $S=\sum_{1}^{n} \frac{1}{N_{i}} \sum_{j \in N_{i}} w_{i j} ; L(G)$ represents the weighting of corporate marketing at keeping unchanged the complex network structure, based on the weighted complex network of corporate marketing degenerate into an average distance not authorized networks $L(G)=\sum_{i, j \in n} d_{i j} / n(n-1)$. The corporate marketing network aggregation degree. $n \geq 2 ; d_{i j}$ indicate the node $i$ and $j$ which to maintain the network structure represents a weighted network structure under the shortest distance between nodes. When $n=1$, so $\partial(G)=1$, apparently $0<\partial(G) \leq 1$.

Based on the above considerations, the importance of the definition of corporate marketing network nodes $v_{i}$ as follows:

$$
\operatorname{NIM}\left(v_{i}\right)=1-\frac{\partial(G)}{\partial\left(G_{v_{i}}^{*}\right)}
$$


Formula (2) further refinement can be obtained:

$$
\operatorname{NIM}\left(v_{i}\right)=\frac{S \times L(G)-S_{v_{i}}^{*} \times L\left(G_{v_{i}}^{*}\right)}{S \times L(G)}
$$

In the formula (3), $G_{v_{i}}^{*} 、 S_{v_{i}}^{*} 、 L\left(G_{v_{i}}^{*}\right)$ respectively, after the new network node $v_{i}$ shrinkage to obtain new networks And the new network a new network to maintain the right of the average point average distance weighted network has no right under the structure of the network.

By the formula (3), the importance of corporate marketing complex network nodes depends on two factors: one is the (number of other network members, namely its product supply and trading and information exchange occurred) nodes connected thereto and tightness (by the close of trading volume and its product supply and demand transaction occurs to reflect); the other is the location of the nodes in the network. Under the same conditions, the greater the degree of the node, the connection between the nodes more closely, the fewer the number of network nodes after contraction, the greater the degree of aggregation network, the more important the node. In addition, if a node in a "critical" position, the shortest marketing channel marketing network impact on the business will be great changes in the average distance of the complex network of corporate marketing, the greater contraction, so corporate marketing degree of aggregation of complex networks will greater. Through the corporate marketing complex network topology using the above method node shrink, the importance of the results of corporate marketing network Wang Yuan judgment on the same structure to assess the importance of an intuitive node is consistent [8].

Based on the above analysis, we give static assess the importance of corporate marketing complex network node algorithm:

(1) Calculate $S$ which the complex network of corporate marketing point average and the right of all nodes;

(2) to maintain basic weighted complex network of corporate marketing structure, it will degenerate into a complex network of corporate marketing network not authorized to calculate the shortest distance between all nodes of the matrix $D(G)=\left[d_{i j}\right]$;

(3) compute weighted aggregation $\partial(G)$ of nodes shrink before corporate marketing of complex networks;

(4) Calculate $S=\left[s_{i j}\right]$ the average node shrink enterprise marketing point right complex array of networks ;

(5) compute nodes shrink not entitled to a complex network of corporate marketing shortest distance between pairs of nodes matrix $D\left(G_{v_{i}}^{*}\right)=\left[d_{i j}\right]$;

(6) FOR $i=1$ to $n / *$ evaluate all nodes in a complex network of corporate marketing importance $* /$

\{

Cohesion $\partial\left(G_{v_{i}}^{*}\right)$ of corporate marketing complex network computing nodes after shrinkage;

To computing nodes importance $\operatorname{NIM}\left(v_{i}\right)$;

\}

\section{Examples Analysis}

Suppose a business marketing network has 10 network members, each network members abstract node. If there is product supply and demand trading relationship between the two Wang Yuan, Wang Yuan then establish a connection between the two sides, trade volume between the two nodes figures represent the edge, resulting in the topology of the enterprise network marketing As shown in Figure 1. Now we come to assess the importance of the static case node. The importance of corporate marketing network node according to the static evaluation algorithm, calculation of the structure of corporate marketing complex network related parameters. 


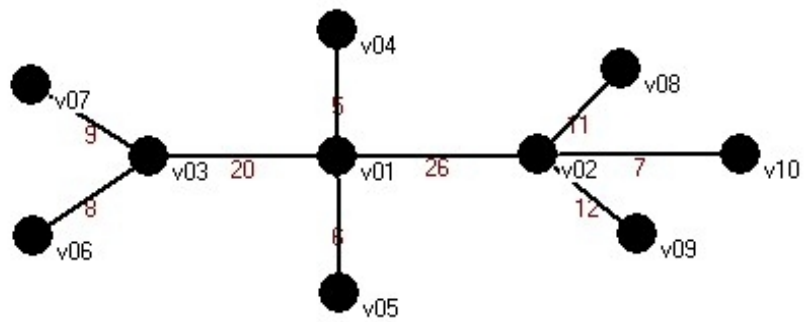

Figure 1 Enterprise marketing network topology diagram

The average point and the right of all nodes as follows:

$$
S=\sum_{1}^{n} \frac{1}{N_{i}} \sum_{j \in N_{i}} w_{i j}=98.58
$$

Weighted basis to maintain corporate marketing complex network structure, based on the above-mentioned complex network of corporate marketing degenerate into unweighted network, calculate the shortest distance matrix between all the nodes .

$$
D(G)=\left[d_{i j}\right]=\left[\begin{array}{cccccccccc}
0 & 1 & 1 & 1 & 1 & 2 & 2 & 2 & 2 & 2 \\
1 & 0 & 2 & 2 & 2 & 3 & 3 & 1 & 1 & 1 \\
1 & 2 & 0 & 2 & 2 & 1 & 1 & 3 & 3 & 3 \\
1 & 2 & 2 & 0 & 2 & 3 & 3 & 3 & 3 & 3 \\
1 & 2 & 2 & 2 & 0 & 3 & 3 & 3 & 3 & 3 \\
2 & 3 & 1 & 3 & 3 & 0 & 2 & 4 & 4 & 4 \\
2 & 3 & 1 & 3 & 3 & 2 & 0 & 4 & 4 & 4 \\
2 & 1 & 3 & 3 & 3 & 4 & 4 & 0 & 2 & 2 \\
2 & 1 & 3 & 3 & 3 & 4 & 4 & 2 & 0 & 2 \\
2 & 1 & 3 & 3 & 3 & 4 & 4 & 2 & 2 & 0
\end{array}\right]
$$

Calculate the start of corporate marketing complex network aggregation degree:

$$
\partial(G)=\frac{1}{S \times L(G)} \approx 0.0029
$$

After the node $v 01$ shrink, network nodes $v 01^{*}$ (node $v 01$ shrink after node $v 01^{*}$ instead of $v 01 、 v 02 、 v 03 、 v 04 、 v 05$ this five nodes) 、 v06、v07、v08、v09、v10 this six nodes, after shrinking network consists of nodes $n=6$. Calculate the average point and the right of all nodes, and kept at a weighted network structure, we have no right to the minimum distance matrix between all the nodes, calculated as follows: $S_{v 01}^{*}=\sum_{1}^{n} \frac{1}{N_{i}} \sum_{j \in N_{i}} w_{i j} \approx 17.33$

$$
D\left(G_{v 01}^{*}\right)=\left[\begin{array}{cccccc}
0 & 1 & 1 & 1 & 1 & 1 \\
1 & 0 & 2 & 2 & 2 & 2 \\
1 & 2 & 0 & 2 & 2 & 2 \\
1 & 2 & 2 & 0 & 2 & 2 \\
1 & 2 & 2 & 2 & 0 & 2 \\
1 & 2 & 2 & 2 & 2 & 0
\end{array}\right]
$$


After the node $v 01$ shrink compute complex network of corporate marketing degree of aggregation:

$$
\partial\left(G_{v 01}^{*}\right)=\frac{1}{S_{v 01}^{*} \times L\left(G_{v 01}^{*}\right)} \approx 0.0346
$$

The importance of nodes $v 01$ :

$$
N I M(v 01)=1-\frac{\partial(G)}{\partial\left(G_{v 01}^{*}\right)}=1-\frac{0.0029}{0.0346}=0.9162
$$

Similarly, the nodes $v 02 、 v 03 、 v 04 、 v 05 、 v 06 、 v 07 、 v 08 、 v 09 、 v 10$ are calculated after shrinking network node degree of aggregation.

For example, given the present corporate marketing network operators, in order of importance to assess the resulting static network members are: $v 01, v 02, v 03, v 09, v 08, v 05, v 04$, $v 07, v 06, v 10$.Assessment results for intuitive judgments, demonstrate the effectiveness of the assessment methodology.

\section{Summary}

In business marketing network topology awareness, based on the analysis of the corporate marketing networks face uncertainty, strengthen the important nodes in the network marketing business protection, whether corporate marketing network can normally play a decisive factor in its product marketing functions. In this paper, corporate marketing network business marketing network node importance evaluation methods and verified by an example of its effectiveness.

\section{References}

[1] High Yunlong. design and management of enterprise marketing network [M] Beijing: Social Science Science Literature Publishing House, 2006.1-4.

[2] Lichao. study has local-world complex networks [D]. Hefei, Anhui University, 2010.

[3] Wurun Ze. Triangle Module node importance evaluation method [J] power fiber optic network based on the North China Electric Power University, 2011, 38 (4): 35-38. [4] Wang Qian. operations research linear programming [J] Jiamusi University Natural Science, 2011,9 (2): 34-37 ..

[5] Wang Xiaofan, Li Xian, Chen Guanrong. complex network theory and its application [M] Beijing: Tsinghua University Press, 2006.18-46.

[6] Wu Jun etc. Given the complex cascade failure load network node important degree assessment [J]. Computer Systems, 2007 (4), 83-86.

[7] Zhu Tao etc. network-based command and control center modeling assessment study [J] System Simulation, 2010 (1): 45-49.

[8] Pastor-Satorras R, Vespignani A. Epidemic spreading in scale-free networks[J]. Physical Review Letters, 2001, 86:3200-3203.

[9] Moore C. Ncwman M E J. Epidemics and percolation in the srnall-world networks. Phys Rev E.2000,61:5678-5682

[10] A.Grabowski and R. A. Kosinski , Epidemic spreading in a hierarchical social network,Phys.Rev.E.2004,70:031908. 